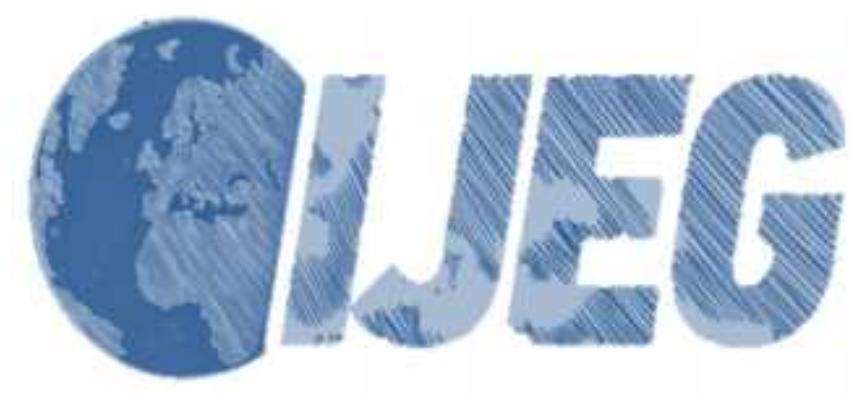

International Journal of Engineering and Geosciences (IJEG),

Vol; 3; , Issue; 1, pp. 006-011, February, 2018, ISSN 2548-0960, Turkey

DOI: $10.26833 /$ ijeg.328919

\title{
PHOTOGRAMMETRIC MODELLING OF HASBEY DAR'ÜLHUFFAZ (MASJID) USING AN UNMANNED AERIAL VEHICLE
}

\author{
Şasi, A., ${ }^{1 *}$ Yakar M., \\ ${ }^{1}$ Selçuk University, Faculty of Engineering, Department of Geomatics Engineering, Konya, Turkey \\ (sasiahmet@gmail.com) \\ ${ }^{2}$ Mersin University, Faculty of Engineering, Department of Geomatics Engineering, Mersin, Turkey \\ (myakar@mersin.edu.tr)
}

ORCID 0000-0002-1217-3887; ORCID 0000-0002-2664-6251

*Corresponding Author, Received:17/07/2017, Accepted: 06/09/2017

\begin{abstract}
Cultural assets constitute the bridge between our past and future in the geography we live. Protecting the artifacts that survived until today and bear the experiences of history and, more importantly, passing these down to future generations are of great importance for our country. Protecting and sustaining cultural assets are duties of mankind for the sake of ownership of universal values. Therefore, documenting all cultural assets in the geography we live in is a necessity. To this end, documentation practices for cultural assets bear great significance in terms of both historical development and contributing to the world cultural heritage.

This article discusses the 3D photogrammetric modelling of Hasbey Dar'ülhuffaz (madrasa for training hafiz) from the Karamanids era, which is located in Ayine Street, Mücellit Neighborhood, Meram District in Konya Province. Within this scope, firstly, four control points that cover the aforementioned cultural asset were established in the study. The coordinates for these points were determined with a Topcon FC-250 GPS. Then, photographs of the cultural asset were captured with Nikon D90 camera and aerial photographs were taken with DJI Phantom 4 unmanned aerial vehicle. DJI Go 4 and Pix4Dcapture mobile applications were used to capture photographs using the unmanned aerial vehicle. All data obtained were evaluated via Agisoft PhotoScan and Netcad software, and a 3D model of the artifact was acquired.

Documentation of the 3D models for artifacts in question contribute greatly to the world cultural heritage. At the same time, the 3D model obtained constitutes a substantial and accurate resource to pass down the artifacts to future generations. The most important point is to provide an opportunity for a faithful restoration in case of any potential damage to this historical artifact from physical intervention and natural disasters. It is foreseen that this model will also offer solutions to the problems of many professional disciplines today.
\end{abstract}

Keywords: Unmanned Aerial Vehicle, 3D Modelling, Photogrammetry, Historical Artifact, Cultural Heritage 


\section{INTRODUCTION}

Historical artifacts that stand as cultural heritage are buildings that connect the past and future of the world. The geography we live in has been home to various cultures and civilizations. Societies belonging to these cultures and civilizations built many assets that reflect their lives and experiences. Thus, it is our duty to display ownership for the cultural assets in our country and sustain them for the sake of history and as a sign of our fidelity. Documentation of cultural assets bearing historical value in our country carries great significance in that the documentation practice helps protect and sustain these structures (Yakar et al., 2011).

The said structures are at risk of being destroyed by natural or unnatural physical interventions at any moment. To this date, countries having cultural and natural heritage have fallen short of protecting such heritage due to a lack of economic, scientific and technical resources. Therefore, it is a national duty of humanity to share scientific studies and recent techniques for the protection of such cultural heritage (Yakar et al., 2009).

Artifacts that stand as cultural assets have detailed and elaborative architectures. For this reason, digital photogrammetry technique is used for the documentation of such cultural assets. Digital photogrammetry technique provides us with the fastest, safest and most accurate data. This technique also employs the state-of-the-art measuring instruments and imaging systems the technology offers us.

In this study, both aerial photogrammetry and terrestrial photogrammetry techniques were used. Four control points that cover the periphery of the cultural asset to be modelled 3D were established in the study. Field coordinates of these points were measured with a GPS. Photographs of the cultural asset were captured using an unmanned aerial vehicle and a camera. Aerial photographs and terrestrial photographs were linked together through tie points.

The 3D model can be used by many professional disciplines. It can be a resource for the historical artifact in order to come up with solutions to problems that may occur in the future. Moreover, thanks to the technological devices used in aerial photogrammetry, the technique of this study establishes a link between history and technology.

\section{PROTECTION AND DOCUMENTATION OF CULTURAL ASSETS}

Documentation procedures for cultural assets having historical property in our country is of great importance. Artifacts can be restored thanks to these documentation procedures in the aftermath of possible damages due to natural disasters (Hanke et al., 2002). Meanwhile, these models can give people an idea in the future, should there be a need for sustaining the original structure of the artifact. They give future generations the correct information regarding the introduction of the past societies (Uysal et al., 2013).

The 3D models obtained via photogrammetry technique constitute an accurate point of reference for administrators of the city and the country alike, facilitating their decision-making process.

\section{FIELD OF STUDY}

\subsection{Hasbey Dar'ülhuffaz}

The name of the cultural asset to be modelled 3D is Hasbey Dar'ülhuffaz (Masjid). It is located in No: 2 Ayine Street, Mücellit Neighborhood, Meram District in Konya Province. The coordinates of Hasbey Dar'ülhuffaz, a cultural asset, are $37^{\circ} 52^{\prime} 90.46^{\prime \prime} \mathrm{N}$ latitude and $32^{\circ} 29^{\prime} 42.42^{\prime \prime} \mathrm{E}$ longitude. It was built in 1421 A.D. during the Karamanids era by Mehmet, the son of Hodja Hasbey, as a madrasa for the hafiz. The artifact was registered by Konya Regional Board of Cultural Heritage Protection on November 13, 1982 (Gümüş et al., 2010).

The artifact is made up of bricks and stones. Above the entrance door hangs an inscription with two lines. The west facade of the structure, which has a square layout, is intensely decorated. The drum of the masjid is covered with a dome which is rather high and made of brick. The mihrab is decorated with mosaic tile pieces some parts of which have not survived until today. Moreover, the artifact has an underground tomb (Gümüş et al., 2010).

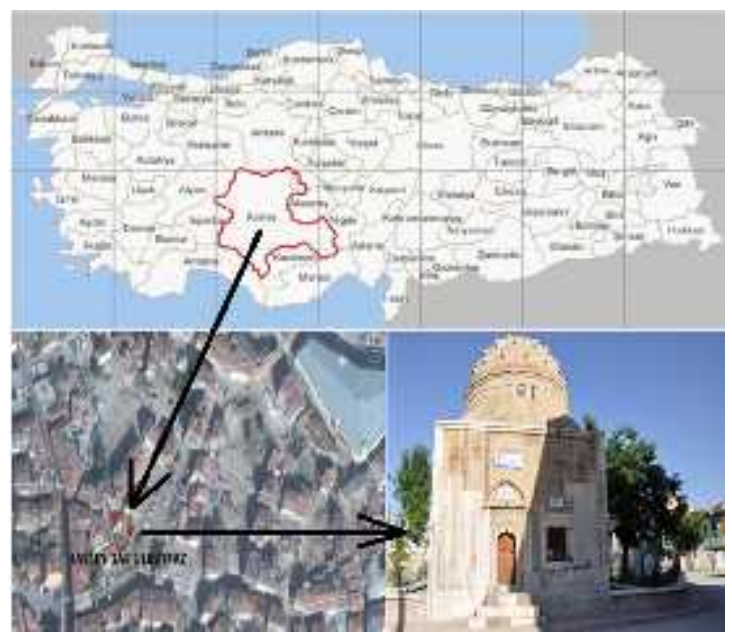

Figure 1. Location and general appearance of Hasbey Dar'ülhuffaz

\subsection{Technical Equipment and Software Used in the Study}

For the 3D modelling of the cultural asset, after establishing four ground control points to cover the periphery of the said asset, coordinates of these points were determined with Topcon FC-250 GPS. A Nikon D90 camera and a DJI Phantom 4 unmanned aerial vehicle were used to take photographs of the historical artifact. For flying the unmanned aerial vehicle, the DJI 
Go 4 mobile application was utilized. Furthermore, weather conditions were monitored via UAV Forecast mobile application to ensure favorable weather conditions were present for flight.

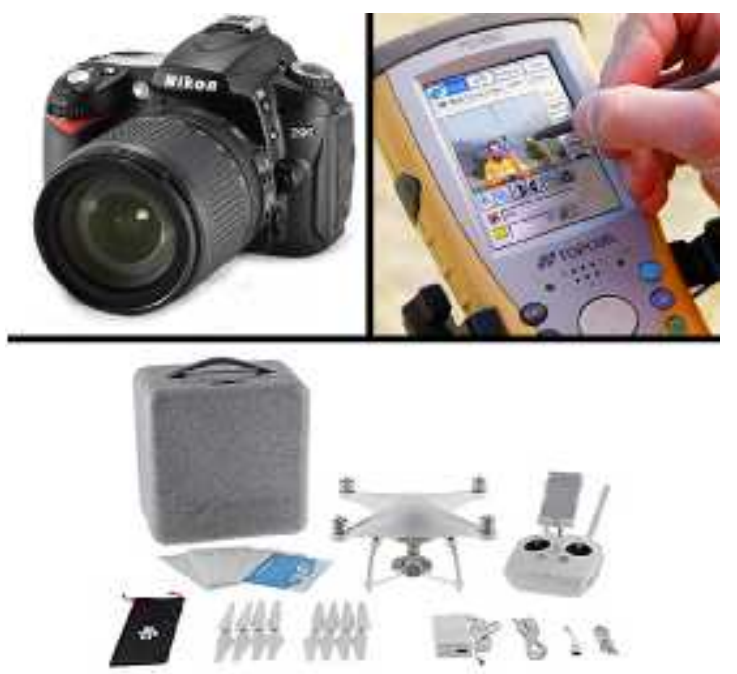

Figure 2. Nikon D90 camera, Topcon FC-250 GPS and DJI Phantom 4 unmanned aerial vehicle

Field coordinates of the ground control points were assessed with Netcad software. Agisoft PhotoScan software was used for the photogrammetric evaluation of the photographs taken.

\subsection{Photogrammetric Evaluation}

The 3D model of the artifact was obtained from the documentation processes for the cultural asset.

In this study, we established four ground control points around the artifact. The field coordinates of these ground control points were measured with Topcon FC250 GPS. Aerial and terrestrial photographs of the artifact were captured using an unmanned aerial vehicle and a camera.

The 3D modelling of Hasbey Darülhuffaz via the photogrammetry technique was carried out in two stages, namely field survey and desktop work. In field surveys, four ground control points were established around the artifact. The coordinates of these points were determined via GPS. The IMU and Compass calibrations for the DJI Phantom 4, the unmanned aerial vehicle, were performed prior to capturing photographs of the artifact. Then, terrestrial and aerial photographs of the artifact were taken with the unmanned aerial vehicle and camera Since the unmanned aerial vehicle used in this study contains a compass, a GPS and two IMUs, the photographs captured with it include latitude, longitude and altitude information. The model which was built by converting coordinates for ground control points established for the field was created with coordinates.

The software used in the evaluation of the photographs of the artifact creates a calibration report of the cameras used. Error corrections are included in the calculation. In order to integrate terrestrial photographs captured with camera and aerial photographs captured with unmanned aerial vehicle, tie points were placed in sharp details among photos. These points were aligned and matched on details that correspond to other photographs. After alignment and matching, the 3D model of the artifact was built via photogrammetric software using all the data obtained from field surveys and desktop work.

\subsubsection{Field Survey for Hasbey Dar'ülhaffaz}

In our country, unmanned aerial vehicles over a certain weight limit must be registered within the system of the Directorate General of Civil Aviation. The unmanned aerial vehicle used in the study was registered before the Directorate General of Civil Aviation. Then, a flight permit was obtained from Konya Governor's Office, and the district police department was provided with information on the location and time of the flight. All these processes are extremely important in terms of public safety as well as flight safety. After the flight permit was obtained and relevant departments were notified, conditions for a safe flight were ensured.

Four control points were established around the artifact to be modelled after the necessary flight permits were obtained. While establishing these points, attention was paid so that points saw one another and nothing obscured the aerial photographs.

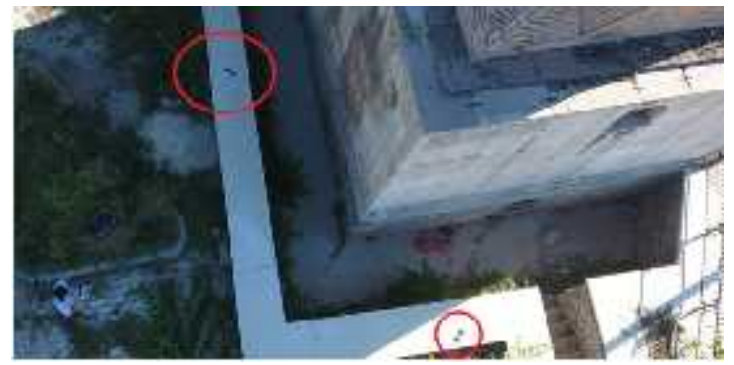

Figure 3. Establishing ground control points

Four control points were established around the artifact to be modelled. Coordinates for these points were determined with Topcon FC-250 GPS and ITRF96 (Epok: 2005). Moreover, the base map of the artifact was taken from the field and vector drawing of it was made via Netcad software.

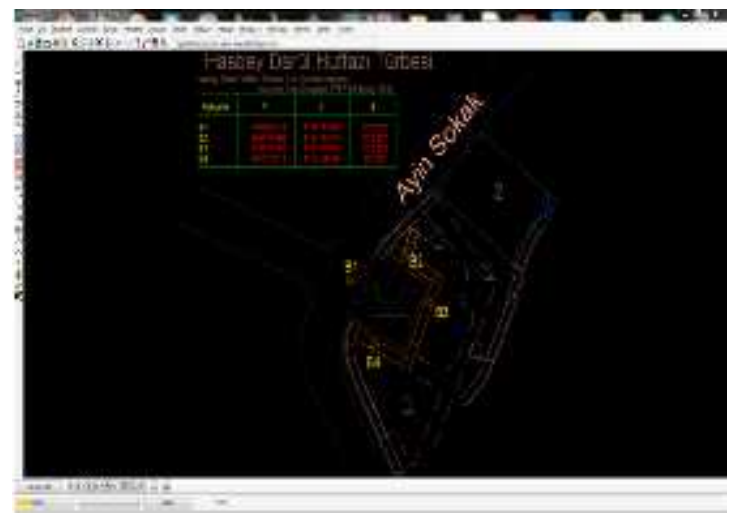

Figure 4. The base map drawing for the artifact 
The UAV Forecast application was used to determine whether the flight zone was fit to fly the unmanned aerial vehicle. This application utilized the information regarding sunrise and sunset time, the speed and direction of the wind, if available, whether the weather is overcast as well as sight distance to determine whether it was a day fit to fly, which it was.

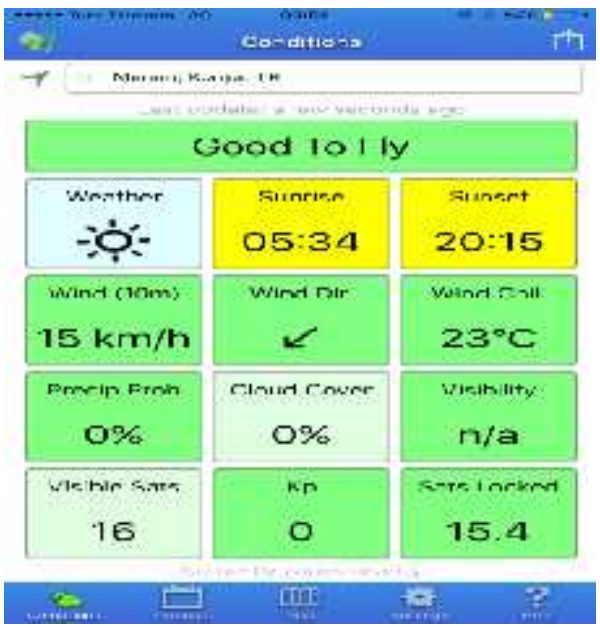

Figure 5. Checking weather conditions for flight

After making sure that the weather conditions were fit for flight, the DJI Phantom 4 and DJI Go 4 mobile application were integrated. Then, the compass of the unmanned aerial vehicle was calibrated. This calibration is important as it eliminates certain system errors that occur while the unmanned aerial vehicle is capturing photographs. The key aspect of the calibration is that while taking photographs, it can ensure the unmanned aerial vehicle vertically suspends in the air in a stable manner.

The Compass is calibrated by rotating the unmanned aerial vehicle $360^{\circ}$ around its own axis both horizontally and vertically.

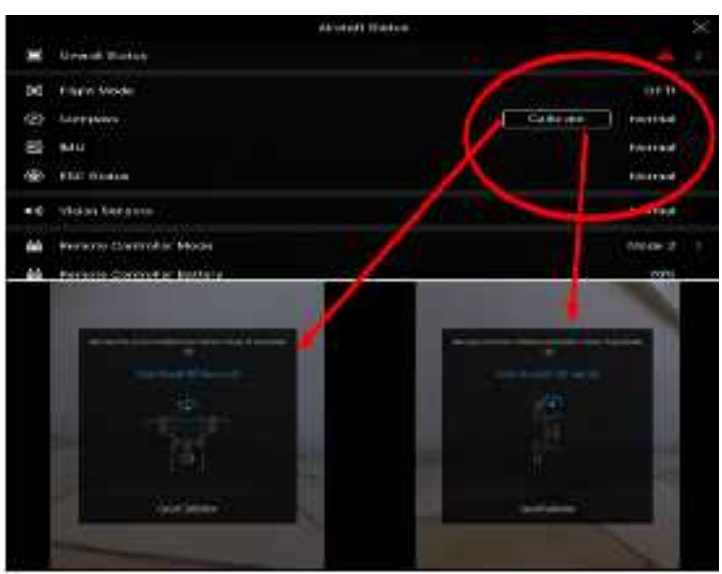

Figure 6. Calibrating the compass of the unmanned aerial vehicle

The compass calibration was followed by the calibration of the IMU. The IMU helps stabilize the unmanned aerial vehicle especially in windy weather conditions and eliminates drift errors in photographs. The calibration process took approximately five minutes.

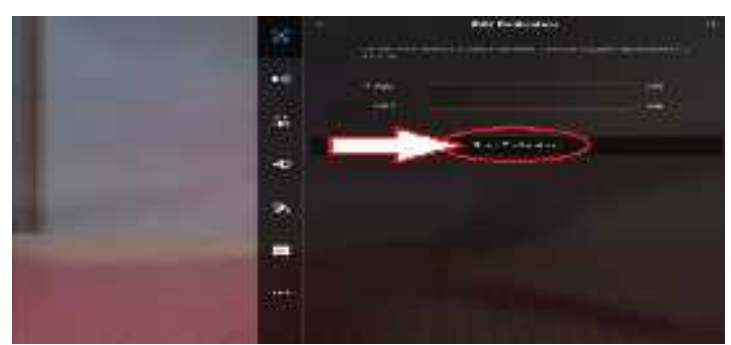

Figure 7. Calibrating the IMU of the unmanned aerial vehicle

Photographs of Hasbey Dar'ülhuffaz were manually controlled by the unmanned aerial vehicle. Each facade of the cultural heritage was photographed, ensuring a high overlapping rate. Aerial photographs were supported by terrestrial photographs taken with the Nikon D90 camera.

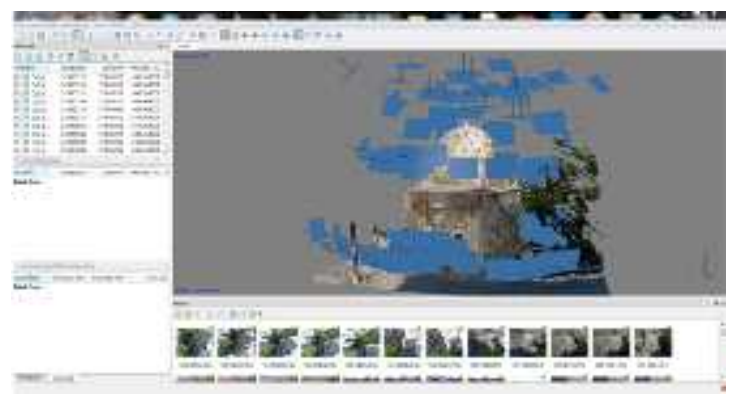

Figure 8. Overlapping photographs of the artifact

The accuracy of geographical coordinates of the cultural asset photographs which were taken by the unmanned aerial vehicle was checked via Google Earth.

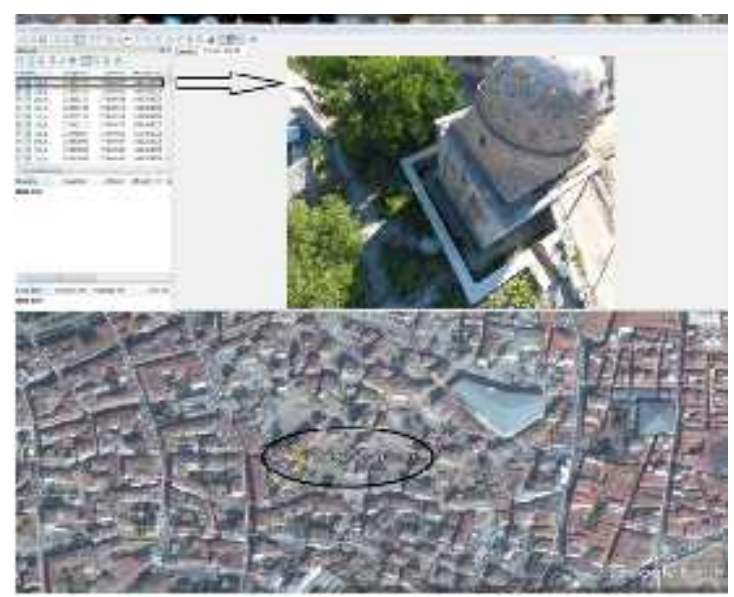

Figure 9. Consistency of photograph and satellite coordinates

Field surveys for Hasbey Dar'ülhuffaz were thus completed. The next stage involved the desktop work. 


\subsubsection{Desktop Work for Hasbey Dar'ülhuffaz}

Photographs of Hasbey Dar'ülhuffaz were evaluated via Agisoft PhotoScan software. First, the photographs were aligned with a high overlapping rate and organized in a certain order. As a result of the alignment, 26,638 point clouds were generated. The process took 4 hours and 22 minutes.

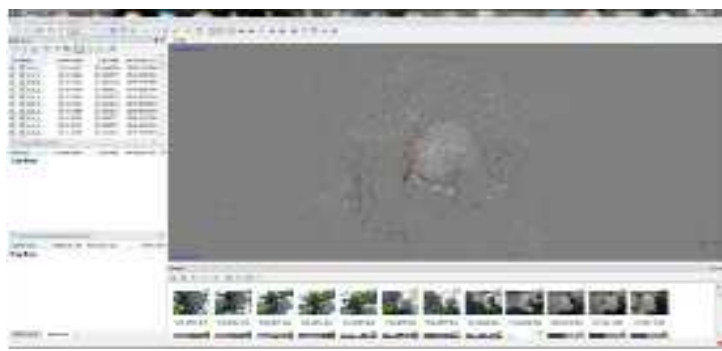

Figure 10. Alignment of Hasbey Dar'ülhuffaz photographs

The aligned photographs helped acquire a point cloud via the high overlapping rate. The second step was to build a dense point cloud. Workflow - Build Dense Cloud - High - Mild option sequence was followed. This process took 7 hours and 23 minutes. As a result, $16,261,059$ point clouds were generated.

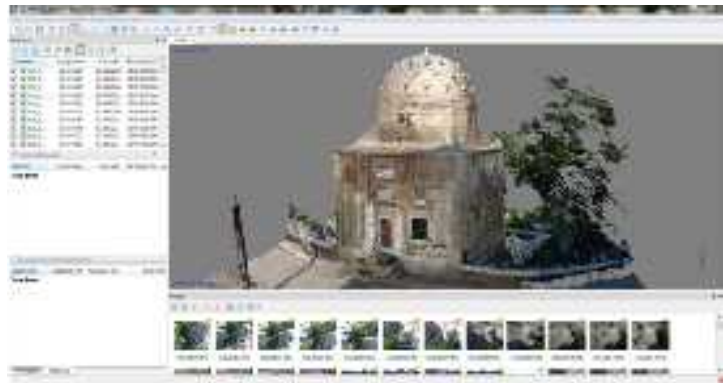

Figure 11. Building a dense cloud for Hasbey Dar'ülhuffaz

Following the completion of building the dense cloud, Workflow - Build Mesh option sequence was followed in order to create a solid model of the dense cloud. Generally, in building modelling, Arbitrary - Dense Cloud - High option sequence was followed and a solid model was obtained by choosing the Enabled option under the Interpolation tab.

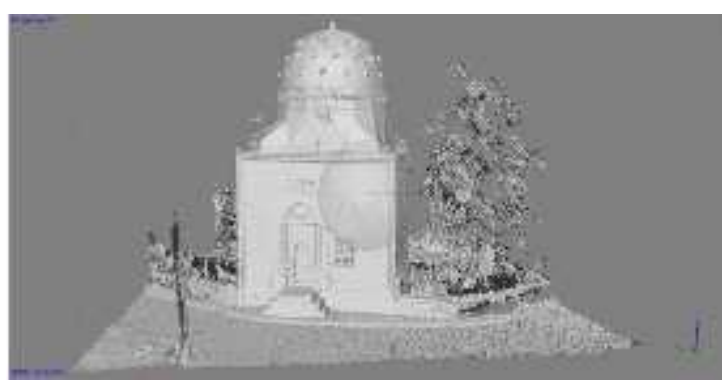

Figure 12. Creating a solid model from point cloud in Build Mesh option
Following the Build Mesh process, Workflow - Build Texture option sequence enabled texture building. In this study, Adaptive Orthophoto (used for vertical texture building) - Mosaic and Enable Color Correction options were used. Following the process, texture building for the solid model was completed.

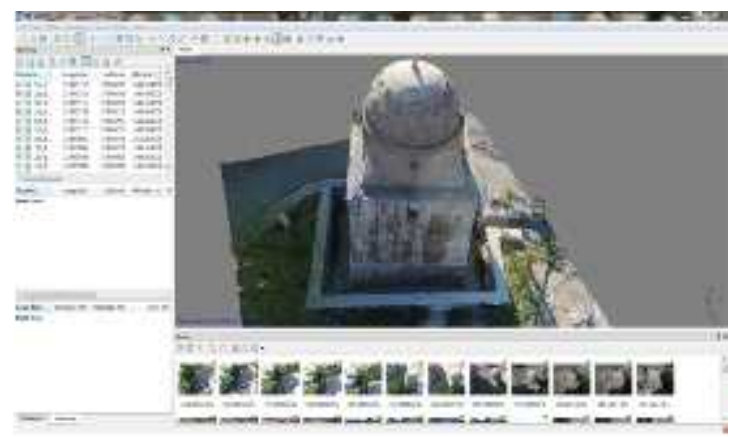

Figure 13. Texture building for the solid model

In order to obtain a high-quality look for the details and the meshes of the object, the Build Tiled Model option was chosen from the Workflow menu. The 3D model of Hasbey Dar'ülhuffaz then included coordinates.

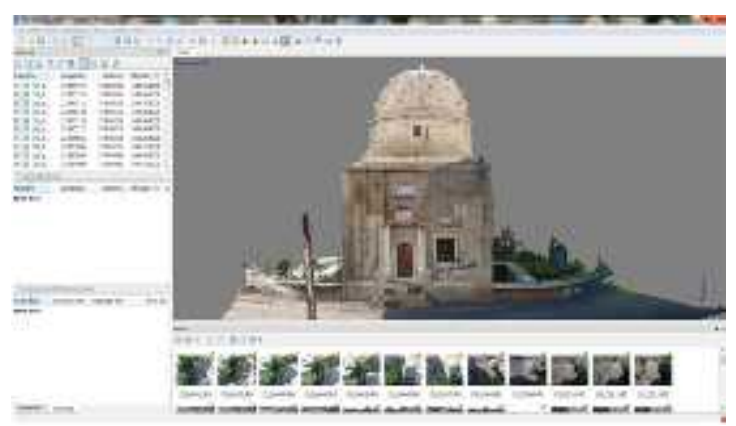

Figure 14. General view of the 3D model (Build Tiled Model)

\section{RESULTS}

As a result of this study, a 3D model of Hasbey Dar'ülhuffaz that is located in Mücellit Neighborhood, Meram District in Konya Province was created via the photogrammetry technique. The 3D model of the artifact was built in two stages, namely field surveys and desktop work. During field surveys, ground control points were established, the coordinates of these points were determined via GPS and the overlapping photographs of the artifact were captured using an unmanned aerial vehicle and a camera. Then, all data obtained from the field were used to build a 3D model during desktop work. Coordinates on the ITRF-96 coordinate system are obtained from the field with a Topcon Fc-250 GPS. The accuracy of the coordinates obtained with the Gps varies between $\pm 2 \mathrm{~cm}$ and $\pm 4 \mathrm{~cm}$.

The 3D model contributed to both the documentation processes for cultural assets of our country and the world cultural heritage. It provides a resource for faithful restoration of the artifact in case of any damage or destruction due to possible natural 
disasters or physical interventions.

The aim of this study is that it constitutes a resource for solving the potential problems of many professional disciplines that may occur around the region in the future.

\section{ACKNOWLEDGEMENTS}

I would like to thank Prof. Murat YAKAR, my advisor, for sharing his knowledge and experience with me and for encouraging me to be a part of and contribute to documentation studies for cultural assets.

\section{REFERENCES}

Gümüş, H., Koçak, F., 2010. Inventory of Immovable Cultural and Natural Heritage of Konya Province. Konya Metropolitan Municipality, Archive for Immovables.

Hanke, K., Grussenmeyer, P., Streıleın, A., 2002. Digital Photogrammetry, Taylor \& Francis, 300 - 301.

Uysal, M., Toprak, A. S., Polat, N., 2013. 'Three Dimensional Modeling of Afyon Gedik Ahmet Pasha (Imaret) Mosque by Photogrammetric Method"' Turkish National Photogrametry and Remote Sensing Association Symposium, Trabzon

Yakar, M., Yılmaz, H.M., Yildı, F., Zeybek, M., Şentürk, H., Çelik, H., 2009. 3-Dimensional Modeling Study and Animation of Roman Period Works in SilifkeMersin Region, TMMOB Chamber of Surveyors and Cadastre Engineers 12. Turkey Map Scientific and Technical Convention, Ankara.

Yakar, M., Yıldız, F., Zeybek, M., Kocaman, E., Yolcu, M., Filiz, T., 2011. Photogrammetric Modeling of Monuments Eflatunpınar, FIG Working Week 2011 Bridging the Gap between Cultures, Marrakechk, Morocco

Web Map Tile Service, www.agisoft.com [Accessed 25 Jun 2017].

Web Map Tile Service, www.dji.com [Accessed 25 Jun 2017].

Web Map Tile Service, https://pix4d.com/ [Accessed 25 Jun 2017].

Web Map Tile Service, https://netcad.com.tr [Accessed 30 Jun 2017].

Copyright (C) International Journal of Engineering and Geosciences (IJEG). All rights reserved, including the making of copies unless permission is obtained from the copyright proprietors. 